\title{
MATERIALS OF A MINI-SYMPOSIUM "NEW TRENDS IN CANCER RESEARCH AND INNOVATIVE TUMOR VACCINES" HELD ON MAY 11, 2017 DURING THE VACTRAIN SUMMER SCHOOL IN KYIV, UKRAINE
}

A mini-symposium "New Trends in Cancer Research and Innovative Tumor Vaccines" was held on May, 11 of 2017 during the VACTRAIN summer school at R.E. Kavetsky Institute of Experimental Pathology, Oncology and Radiobiology (IEPOR) of the NAMS of Ukraine, Kyiv, Ukraine.

On this day, young researchers have presented results of their current work, along with few keynote lectures. The main focus of this event was to understand the modern trends to find the new markers to diagnose cancer disease and new targets for cancer vaccines. Also, there was a discussion on novel cancer vaccines.

The deputy director of IEPOR Associated professor Lubov Buchynska welcomed the participants and wished the fruitful discussion, concerning new approaches in the development of cancer vaccines. Professor Chanchal Mitra (Hyderabad University, India) gave a wide overview on miRNAs that play an important role in cancer development. Associated professor Elena Kashuba (IEPOR) presented the new data on a role of mitochondrial ribosomal protein S18-2 as the new oncoprotein and a putative target for cancer vaccine. More data and the future perspectives are described in abstracts of young researchers from Ukraine, Belarus, Latvia, and Lithuania (see below).

Concluding mini-symposium, supported by the VACTRAIN of Horizon 2020 program, Elena Kashuba thank VACTRAIN for the possibility of schooling the young researchers with an aim to help them to became the critically thinking open-minded researchers. The importance of scientific collaboration was also enlightened. Definitely, this summer school resulted in the enhanced knowledge in medical science and helped to plan the new fruitful scientific works with the aim to combat cancer.

\section{SELECTED ABSTRACTS}

\section{COMPARISON OF DIFFERENT KIND OF DENDRIMERS FOR THE ANTICANCER RNAi GUIDES DELIVERY \\ Volha Dzmitruk', Maksim lonov', Dzmitry Shcharbin', Maria Bryszewska ${ }^{2}$}

${ }^{1}$ Institute of Biophysics and Cell Engineering of NAS of Belarus, Minsk, Belarus

${ }^{2}$ University of Lodz, Lodz, Poland

E-mail:dmitruk.olga@gmail.com

One of the directions of gene therapy is curative cancer treatment. During transformation, normal cells start to propagate uncontrollably and lose the ability to undergo apoptosis, resulting in tumor formation. Expression of apoptosis inhibitors (BCL-2 family proteins) can be downregulated at a post-translational level by means of RNA interference (RNAi), i.e. by a process of highly selective gene silencing. RNAi effectors - siRNA (small interfering RNA) or microRNA are able to cleave mRNA, using the cellular machinery (Burlacu, 2003). The major limit of such gene therapy application is an absence of effective delivery of nucleic acids (siRNA) into the target cells (Songet et al., 2005).

Dendrimers are synthetic polymers, highlighted for gene delivery, due to their monodispersity, predetermined tree-like structure, stability, low viscosity of their solutions and a large number of charged end groups. Cationic dendrimers can complex with nucleic acids by self-assembly, making complexes called "dendriplexes" (Wang et al., 2010).

Different kinds of dendrimers were tested as delivery systems for different RNAi effectors. It was revealed that PAMAM (carbosilane and phosphorous-containing dendrimers) form stable complexes with small acting RNAs by self-assembling in up to $1 \mu \mathrm{m}$ in size. In contrast to others, phosphorous dendrimers are insensitive to heparin impact, thus indicating not only electrostatic interaction within the dendriplex (lonov et al., 2015). Cytotoxicity studies on HeLa (cervical cancer) and HL-60 (promyelocytic T-leukemia) cell lines showed the synergism in action of siRNAs specific for various anti-apoptotic proteins, in comparison with treatment with each single siRNA. This was observed when siRNAs were delivered by cationic dendrimers. In our experiments, the cytotoxicity of siRNA/dendrimer complexes showed a dual nature, based on three mechanisms: apoptosis, autophagy, and necrosis. The siRNA induced apoptosis and, moreover, dendrimers themselves may induce all above, depending on the chemical nature of dendrimer (Dzmitruk et al., 2015).

This work was supported by 2 grants from the Belarusian Republican Foundation for Fundamental Research No. B15RM-060 and No. B15CO-041, a Marie Curie International Research Staff Exchange Scheme Fellowship within the $7^{\text {th }}$ European Community Framework Programme, project No. PIRSESGA-2012-316730 NANOGENE. 
SIMULTANEOUS CD150 AND CD180 LIGATION MUTUALLY INHIBIT AKT AND MAPK SIGNAL TRANSDUCTION PATHWAYS IN THE CHRONIC LYMPHOCYTIC LEUKEMIA B CELLS

Inna Gorditienko, Larysa Shlapatska, Valeriia Kholodniuk, Lilia Sklyarenko, Svitlana Sidorenko

R.E. Kavetsky Institute of Experimental Pathology, Oncology and Radiobiology, NAS of Ukraine, Kyiv, Ukraine

E-mail:imgordiienko@gmail.com

Combination of input signals from cell surface receptors (e.g. BCR, CD140, BAFFR, TLR) in B-cell malignancies underlies disease outcome. It was shown that cell surface CD150 (csCD150) and CD180 (csCD180) expression in chronic lymphocytic leukemia (CLL) correlates with favourable prognosis.

We found that csCD150 expression has strong positive correlation with csCD180 expression in CLL cases $(r=$ $0.75, p=0.0001)$. Moreover, CD150 showed the highest level of colocalization with $\operatorname{CD} 180(R=0.88 \pm 0.13 ; R[r]=$ $0.87 \pm 0.1)$ at a cell membrane of CLLB cells. To determine the biological significance of simultaneous CD150 and CD180 expression in CLL pathobiology we performed in vitro stimulation assay followed by signaling pathways analysis. CD150 or CD180 crosslinking on CLL B cells alone resulted in activation of Akt, mTORC1, ERK1/2, p38MAPK and JNK1/2 pathways. Both CD150 and CD180 receptors affect protein synthesis through p70S6K phosphorylation and transmit pro-survival signals via Akt-mediated GSK3 $\beta$ and FOXO1/FOXO3a transcription factors inhibition. It was surprising that simultaneous CD150 and CD180 ligation blocked Akt, mTORC1 as well as MAPK pathways activation in CLL B cells. Moreover, in the absence of CD150 and CD180 on cell surface, both antigens were expressed on the protein level in the cytoplasm. Intracellular distribution of CD150 and CD180 in CLLB cells showed different pattern. CD150 antigen was colocalized with markers of endoplasmic reticulum, Golgi apparatus, early endosomes, but only trace amount was detected in lysosomes. In contrast, CD180 was detected preferentially in early endosomes $(R=0.60 \pm 0.10 ; R[r]=0.59 \pm 0.10)$. It could be concluded that malignant CLL B cells try to escape simultaneous cell surface CD150 and CD180 expression since combined ligation of these receptors leads to blocking of pro-survival pathways.

\section{ADAPTOR PROTEIN RUK/CIN85 INCREASES TUMORIGENICITY OF MAMMARY ADENOCARCINOMA CELLS VIA PROMOTING EMT}

Iryna Horak, Ganna Pasichnyk, Dmytro Shytikov, Denys Geraschenko, Liudmyla Drobot

Palladin Institute of Biochemistry, NAS of Ukraine, Kyiv, Ukraine

E-mail: iryna.horak@gmail.com

Adaptor proteins serve as molecular platforms for multimolecular complexes assembly and thereby regulate intracellular signaling. Our previous results demonstrated that tumors (including breast cancer) are characterized by increased expression of adaptor protein Ruk/
CIN85 in comparison to normal tissues. This adaptor plays a critical role in several cellular processes, such as ligandinduced endocytosis of RTKs, intracellular vesicular trafficking, adhesion, motility, and survival. In this study we investigated the effect of Ruk/CIN85 on tumorigenicity of mammary adenocarcinoma cells.

As a model we used mouse 4T1 mammary adenocarcinoma cells with stable overexpression (RukUp) and downregulation (RukDown) of Ruk/CIN85. Motility and invasiveness of these cells were analyzed by scratch test and Boyden chamber assay. The effectiveness of metastasis formation was studied using experimental metastasis assay. qPCR andWestern blotting were carried out to evaluate gene and protein expression.

We demonstrated that Ruk/CIN85 overexpression leads to elevation of motility and invasiveness in vitro and increased the ability to produce lung metastasis in vivo. Also, we demonstrated increased expression of mesenchymal markers in RukUp cells and epithelial markers in RukDown cells that mean Ruk/CIN85 promotes EMT in 4T1 cells.

\section{IDENTIFICATION OF NOVEL MOLECULAR AND GENETIC MARKERS FOR EARLY DETECTION OF EPITHELIAL TUMORS} AND PROGNOSIS OF THE COURSE OF DISEASE

Oksana Mankovska, Vladimir Kashuba

Institute of Molecular Biology and Genetics, NAS of Ukraine, Kyiv, Ukraine

E-mail: mankovsska@gmail.com

The main topic of our research is the identification of molecular and genetic markers for the early detection of epithelial tumors and prognosis of the disease. Early detection of cancer can lead to more successful treatment, thus, significantly increasing the chances for recovery. The investigation of non-invasive diagnostic tools, which can be used in clinical practice, is now the main focus of oncology. This problem can be solved by using of molecular nucleic acid markers that are present in biological fluids (serum, urine, semen etc).

We have developed several useful and effective approaches for the investigation of molecular and genetic markers. We are studying both, genetic and epigenetic changes in cancers of different types, using a broadscale screening of their presence in clinical samples. The techniques and methods we use allow us to obtain reliable results and propose them for implementation in clinical practice.

\section{DIAGNOSTIC VALUE OF NEUTROPHIL/ LYMPHOCYTE RATIO IN HEPATOCELLULAR CARCINOMA AND FOCAL NODULAR HUPERPLASIA: A PILOT STUDY}

Dzeina Mezale ${ }^{1}$, Ilze Strumfa', Andrejs Vanags' ${ }^{2}$, Ilze Fridrihsone', Polina Viktorova'

${ }^{1}$ Department of Pathology, Riga Stradins University, Riga, Latvia

${ }^{2}$ Department of Surgery, Riga Stradins University, Riga, Latvia

E-mail: dzeina.mezale@gmail.com

Hepatocellular carcinoma (HCC) is the sixth most common malignancy and the third most common 
cause of cancer death worldwide (Jain et al., 2017). Distinguishing a well-differentiated $\mathrm{HCC}$ from benign liver nodules, such as focal nodular hyperplasia (FNH), may be difficult in some cases, particularly in small needle core biopsies (Coston et al., 2008), thus additional diagnostic markers are essential. Routinely available marker of the systemic inflammatory response such as neutrophil - lymphocyte ratio (NLR) has recently been reported as a predictor of HCC; an elevated level indicating a poor prognosis (Xiao et al., 2014). The aim of the present study was to examine whether NLR could be used as an additional diagnostic marker distinguishing between $\mathrm{HCC}$ and $\mathrm{FNH}$.

In a retrospective pilot study, 14 patients with morphologically confirmed HCC and 6 patients with FNH were included. NLR was defined as the absolute neutrophil count divided by the absolute lymphocyte count. Complete blood count levels were used to calculate NLR. Data were evaluated using IBM SPSSv23. To assess data distribution, an independent-samples Kolmogorov - Smirnov test was performed. Descriptive statistic evaluation was performed, including detection of median values and interquartile range $\mathrm{IQR}=\mathrm{Q}_{3}-\mathrm{Q}_{1}$. To compare groups, independent samples median test and Fisher exact significance test were used. The significance level was set at 0.05 .

The median value of NLR in FNH group was $1.67\left(\mathrm{~N}=6 ; \mathrm{Q}_{1}=1.20 ; \mathrm{Q}_{3}=2.57 ; \mathrm{IQR}=1.37\right)$. The median NLR of the patients with $\mathrm{HCC}$ was $2.49(\mathrm{~N}=14$; $\left.Q_{1}=1.97 ; Q_{3}=4.55 ; I Q R=2.58\right)$, which is higher comparing with $\mathrm{FNH}$ group, but the difference was statistically insignificant $(p=0.141)$.

Concluding, we showed that i) A complete blood count with following calculation of NLR is widely available blood test, which has a potential to be a useful additional marker distinguishing between $\mathrm{HCC}$ and benign liver nodules, such as $\mathrm{FNH}$; ii) The median NLR in HCC patient group was higher than in FNH group (1.67 and 2.49, respectively), which corresponds to the available scientific data, but the difference was found to be statistically insignificant. However, further research in a larger group is clearly required.

\section{INTERPLAY BETWEEN MITOCHONDRIAL RIBOSOMAL PROTEIN S18-2 AND RETINOBLASTOMA SUSCEPTIBILITY (RB) PROTEIN IN REGULATION OF CELL STEMNESS AND DIFFERENTATION \\ Muhammad Mushtaq', Larysa Kovalevska ${ }^{2}$, Elena Kashuba ${ }^{1,2}$ \\ ${ }^{1}$ MTC, Karolinska Institutet, Stockholm, Sweden}

${ }^{2} R$. E. Kavetsky Institute of Experimental Pathology, Oncology and Radiobiology, NAS of Ukraine, Kyiv, Ukraine

E-mail: kreyl@yahoo.com

We have found that S18-2 is involved in regulation of the RB-dependent pathway. It binds to both hypo- and hyperphosphorylated RB. The binding be- tween RB and S18-2 is promoted when cytoplasmic $\mathrm{S} 18-2$ is targeted to the nucleus, and this disrupts the association of E2F1 with $\mathrm{RB}$, as indicated by the increased level of free E2F1 in the nucleus (Kashuba et al., 2008). This presumably lifts the RB-dependent block of $S$ phase entry. We have also found that overexpression of the human S18-2 immortalized primary rat embryonic fibroblasts that showed properties of embryonic stem cells (Kashuba et al., 2009). Elevated expression of S18-2 in stem cells (our findings and analysis of published microarray data) raises the question of whether this protein cooperates with the RB protein in cell differentiation and oncogenesis.

We aimed to seek a connection between the expression of RB and S18-2 in Rb1-/- mouse embryonic fibroblasts and stemness. We hypothesized that simultaneous expression of both proteins at the high levels might support stemness. Transfections, inoculation into SCID mice, directed differentiation, GPCR, immunostaining, immunohistochemistry, Western blotting, zebrafish embryogenesis were used in the present work.

We showed that S18-2 protein, together with $\mathrm{RB}$, plays a crucial role in cell de-differentiation. We have also found that overexpression of S18-2 and $\mathrm{RB}$ is needed for maintenance of cell stemness. Such cells can differentiate into various cell lineages under certain conditions. The presence of RB and simultaneous expression of S18-2 at high levels are required for the cell stemness.

\section{INHIBITION OF IRE1 SIGNALING AFFECTS THE EXPRESSION OF GENES ENCODING GLUCOCORTICOID RECEPTOR AND SOME RELATED PROTEINS IN U87 GLIOMA CELLS Oksana Ratushna, Dmytro Minchenko, Olena Riabovol, Oleksandr Minchenko \\ Palladin Institute of Biochemistry, NAS of Ukraine, Kyiv, Ukraine \\ E-mail: oksana_ratushna@hotmail.com}

Glucocorticoid receptor (nuclear receptor subfamily 3 , group C, member 1; NR3C1) plays an important role in the regulation of numerous metabolic and proliferative processes, including tumorigenesis and metabolic diseases. Regulation of these processes by glucocorticoids is preferentially stress-dependent, but the involvement of the endoplasmic reticulum (ER) stress signaling has not been explored yet.

The aim of the present investigation was to examine the effect of inhibition of ER stress signaling mediated by IRE1 (inositol requiring enzyme 1), which is a central mediator of the unfolded protein response, on the expression of genes encoded NR3C 1 and some related proteins (SGK1, SGK3, NCOA1, NCOA2, ARHGAP35, NRIP1, and NNT) and their hypoxic regulation in U87 glioma cells for evaluation of their possible significance in the control of glioma growth.

The expression of NR3C1, SGK1, SGK3, NCOA1, NCOA2, ARHGAP35, NRIP1, and NNT genes in U87 glioma cells transfected by empty vector pcDNA3.1 (control) 
and cells without IRE1 signaling enzyme function (transfected by dnIRE1) upon hypoxia was studied by qPCR.

We have shown that inhibition of IRE1 signaling enzyme function up-regulates the expression of NR3C1, SGK1, NCOA1, NCOA2, ARHGAP35, and NNT genes in U87 glioma cells in comparison with the control glioma cells, being more significant changes for NR3C1, SGK1, and $N N T$ genes. At the same time, the expression of SGK3 and NRIP1 genes is strongly down-regulated in glioma cells upon inhibition of IRE1. We have also shown that hypoxia increases the expression of $N R 3 C 1$, SGK1, NCOA2, ARHGAP35, and NNT genes but decreases SGK3, NRIP1, and NCOA1 genes expression in control glioma cells. Moreover, the inhibition of both enzymatic activities (kinase and endoribonuclease) of IRE1 in U87 glioma cells enhances the effect of hypoxia on the expression of SGK1, SGK3, and NNT genes but decreases the sensitivity of $N R 3 C 1$ gene to hypoxic condition. Furthermore, the expression of NCOA 1 gene is resistant to hypoxia in control glioma cells, but NCOA2, NRIP1, and ARHGAP35 genes are resistant to this condition in glioma cells without functional activity of IRE1 signaling enzyme.

Results of this investigation demonstrate that inhibition of IRE1 signaling enzyme function affects the expression of NR3C1, SGK1, SGK3, NCOA1, NCOA2, ARHGAP35, NRIP1, and NNT genes in U87 glioma cells in the gene specific manner and that all these genes are regulated by hypoxia preferentially through IRE1 signaling pathway of ER stress.

\section{EXPRESSION OF CANCER-ASSOCIATED GENES IN PROSTATE TUMORS}

\section{Evgenia Rosenberg', Ganna Gerashchenko', Natalia Hryshchenko', Raisa Lytvynenko', Yuriy Vitruk ${ }^{2}$, Oleksandr Gryzodub ${ }^{2}$, Eugenia Stakhovsky ${ }^{2}$, Vladimir Kashuba' 'Institute of Molecular Biology and Genetics, NAS of Ukraine, Kyiv, Ukraine \\ ${ }^{2}$ National Cancer Institute, Ministry of Healthcare of Ukraine, Kyiv, Ukraine \\ E-mail: kalirra@mail.ru}

Despite numerous studies that aimed to define the molecular mechanisms that underlay formation and progression of prostate tumors, markers of advanced prostate cancer are still not elaborated. The objective of our investigation was an analysis of relative gene expression of a set of cancer-associated genes, previously selected based on a work with the prostate cancer cell lines. We studied the following cancer-associated genes in the clinical samples of prostate tumors TGFB1, IL1B, FOS, EFNA5, TAGLN, PLAU, and EPDR1.

To study the expression of the abovementioned genes, a total RNA from 16 prostate adenomas, 37 adenocarcinomas and 29 conventionally normal tissues were isolated. qPCR was performed to analyze the gene expression pattern. Immunohistochemistry was conducted to determine the expression of the TGF $\beta 1$, FOS, TAGLN, and PLAU proteins.
Alterations in the relative gene expression levels were found for TGFB1, IL1B, FOS, EFNA5, TAGLN, $P L A U$, and EPDR1 genes in all analyzed groups. For TGFB1, FOS, TAGLN, and PLAU genes obtained results were confirmed on the protein level. FOS protein was increased during progression from adenoma to aggressive prostate cancer. Meanwhile, levels of TGF $\beta 1$, TAGLN, and PLAU proteins were decreased in advanced prostate cancer.

Concluding, the obtained results might indicate that TGFB1, FOS, TAGLN, and PLAU are potential biomarkers for prostate cancer. Moreover, FOS gene may be a putative target for anti-cancer therapy. However, these results are preliminary and it requires the further investigations.

\section{THE ROLE OF EPIGENETICS IN THE DEVELOPMENT OF TYPE 2 DIABETES AND EFFECTIVENESS OF METFORMIN THERAPY \\ Monta Ustinova, Ineta Kalninga, Ilze Elbere, Janis Klovinš \\ Latvian Biomedical Research and Study Centre, Riga, Latvia \\ E-mail: monta.ustinova@biomed.lu.Iv}

The incidence of type 2 diabetes mellitus (T2D) has become an epidemic, increasing the risk of cardiovascular diseases and premature death. The global T2D prevalence is $8.3 \%$ with the highest burden in developing countries. Metformin is a first-line therapy in T2D treatment, but pharmacodynamics of metformin is still not clear. There is a variable efficiency observed for metformin therapy; furthermore, nearly one-third of T2D patients do not respond to it.

So far, there are no validated biomarkers that would predict the effectiveness of diabetes medications and the patient's responses to treatment. Epigenetic signatures are considered to be promising biomarkers for the evaluation of the therapeutic efficiency in metabolic diseases. The impact of metformin on epigenetic regulation has not been described.

Studies on metformin's mechanism of action and development of new epigenetic biomarkers will allow to predict the effectiveness and the potential toxicity of metformin in T2D patients.

The aim of the study is to determine the epigenetic patterns related with T2D and response to antidiabetic drug therapy. The main objectives are the following: i) To identify metformin-induced novel DNA methylation and RNA expression signatures in healthy individuals and T2D patients; ii) To validate potential epigenetic biomarkers in a large cohort of T2D patients after long-term metformin therapy; iii) To estimate the concordance between epigenetic patterns derived from white blood cells with those from target tissues, by analysis of DNA methylation and RNA expression in mice; and iv) To develop an epigenetic-based algorithm for prediction of efficacy and side-effects of metformin therapy. 